\title{
The Effects of Propofol on Hypoxia- and TNF- $a-$ Mediated Brain-Derived Neurotrophic Factor/Tyrosine Kinase Receptor B Pathway Dysregulation in Primary Rat Hippocampal Neurons and Astrocytes
}

\section{Weiping Tao}

Jing'an District Centre Hospital of Shanghai

Xuesong Zhang

Shanghai Public Health Clinical Center

\section{Juan Ding}

Shanghai Cancer Hospital: Fudan University Shanghai Cancer Center

\section{Shijian Yu}

Jing'an District Centre Hospital of Shanghai

\section{Peiqing Ge}

Jing'an District Centre Hospital of Shanghai

Jingfeng Han

Jing'an District Centre Hospital of Shanghai

Xing Luo

Shanghai Cancer Hospital: Fudan University Shanghai Cancer Center

Wei Cui

Jing'an District Centre Hospital of Shanghai

Jiawei Chen ( $\square$ jiawei_chen@hotmail.com )

Jing'an District Centre Hospital of Shanghai https://orcid.org/0000-0003-3726-9823

\section{Research Article}

Keywords: Astrocyte, Brain-derived neurotrophic factor, Hippocampal neuron, Hypoxia, Tumor necrosis factor-a, Propofol

Posted Date: June 21st, 2021

DOl: https://doi.org/10.21203/rs.3.rs-607889/v1 
License: (c) (i) This work is licensed under a Creative Commons Attribution 4.0 International License. Read Full License 


\section{Abstract}

Background: BDNF/TrkB pathway dysregulation may be induced by hypoxia and inflammation, and play pivotal roles during the development of neurological disorders. Propofol is an anesthetic agent with neuro-protective properties. We aimed to verify whether propofol affected BDNF/TrkB pathway in neurons exposed to hypoxia or TNF-a.

Methods: Primary rat hippocampal neurons and astrocytes were cultured and exposed to propofol followed by hypoxia or TNF-a treatment. The production of BDNF and the expression/truncation/phosphorylation of TrkB were measured. The underlying mechanisms such as ERK, CREB, p35 and Cdk5 were investigated.

Results: In hippocampal neurons and astrocytes, hypoxia and TNF-a reduced the production of BDNF. Pretreatment of hippocampal neurons with $25 \mu \mathrm{M}$ propofol reversed the inhibitory effect of hypoxia or TNF-a on BDNF production. However, even $100 \mu \mathrm{M}$ propofol had no such effect in astrocytes. Further, we found that in hippocampal neurons hypoxia and TNF-a increased the phosphorylaion of ERK (p-ERK) and

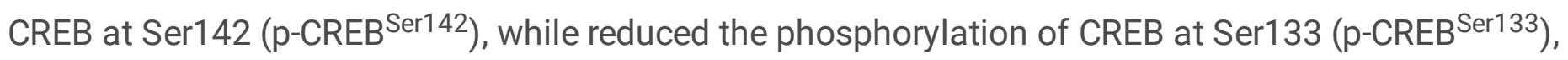
which were all reversed by $25 \mu \mathrm{M}$ propofol and $10 \mu \mathrm{M}$ ERK inhibitor. In addition, neither hypoxia nor TNF-a affected TrkB expression, truncation or phosphorylation in hippocampal neurons and astrocytes. However $50 \mu \mathrm{M}$ propofol induced TrkB phosphorylation without affecting its expression and truncation only in hippocampal neurons. Furthermore, we detected that in hippocampal neurons, 50 $\mathrm{M}$ propofol induced p35 expression and Cdk5 activation, and blockade of p35 or Cdk5 mitigated propofol-induced TrkB phosphorylation.

Conclusions: Propofol, via ERK/CREB and p35/Cdk5, may modulate BDNF/TrkB pathway in hippocampal neurons that were exposed to hypoxia or TNF-a.

\section{Introduction}

Brain-derived neurotrophic factor (BDNF) is one of the most studied and well characterized neurotrophic factors in the central nervous system (CNS), and is mainly produced in the brain by hippocampal neurons and astrocytes. BDNF plays an extensively role in neuronal growth, differentiation, survival, synaptic plasticity and neurotransmitter regulation [1], and therefore has been considered to have potential therapeutic values during the development of neurological disorders, such as cerebral ischemiareperfusion injury [2], neuroinflammation-related brain injury [3], age-related memory impairment [4], Parkinson's disease [5], Alzheimer's disease [6] and postoperative cognitive dysfunction [7]. It was originally thought that BDNF exerts its biological functions through binding to two transmembrane receptors, the tropomyosin receptor tyrosine kinase B (TrkB) and p75 neurotrophin receptor (p75 NTR). However, accumulating evidence suggest that mature BDNF has high affinity to TrkB, while its precursor proBDNF mainly activates p75 NTR [8]. Numerous in vitro and animal studies revealed that multiple pathophysiological stimuli such as oxidative stress, inflammation, and ischemia/reperfusion injury may 
induce damages in the CNS via affecting BDNF/TrkB signaling [9], and recently, BDNF/TrkB signaling has been identified to serve as a potential therapeutic target for depression [10], post-cerebral ischemic spatial cognitive dysfunction [11], vascular dementia [12] and postoperative cognitive dysfunction [13].

Propofol is (2, 6-diisopropyl phenol) is an intravenous general anesthetic, which is extensively used in the induction and maintenance of anesthetization and procedural sedation. Apart from its multiple anesthetic advantages, it has been reported to possess anti-oxidative and anti-inflammatory effects [14, 15] as well as neuro-protective properties [16]. A number of in vitro studies revealed that propofol may protect mouse hippocampal neurons from inflammation-induced autophagy [17] and from inflammationand hypoxia- as well as oxidative stress-induced apoptosis [18-20]. In addition, propofol may protect hypoxia- and inflammation-impaired integrity of blood-brain barrier (BBB) in the in vitro model [17, 21-23]. Although propofol has been reported to modulate the expression of BDNF and TrkB in the hippocampus of aged rats that were exposed to cerebral ischemia injury [24], the effects of propofol on BDNF/TrkB pathway in the neurons, especially those neurons exposed to vicious stimuli such as hypoxia and inflammation, have not been thoroughly investigated.

Therefore, in this study we aimed to detect whether propofol could modulate hypoxia- and TNF-amediated BDNF/TrkB pathway dysregulation in primary rat hippocampal neurons and astrocytes, and further investigated the underlying mechanisms.

\section{Materials And Methods}

\section{Experimental design}

Primary rat hippocampal neurons and astrocytes were cultured in normoxic condition ( $95 \%$ humidified air and $5 \% \mathrm{CO}_{2}$ ) until ready for experiments. To mimic hypoxic condition, cells were maintained in a hypoxic chamber flushed with a humidified gas mixture ( $90 \%$ humidified $\mathrm{N}_{2}, 5 \% \mathrm{O}_{2}$ and $5 \% \mathrm{CO}_{2}$ ) for different times $(0,1,2,3,6,12 \mathrm{~h})$. To mimic inflammation condition, cells were treated with $40 \mathrm{ng} / \mathrm{mL}$ TNF-a for different times $(0,1,2,3,6,12 \mathrm{~h})$. To examine the effect of propofol, cells were treated with different concentrations $(1,5,10,25,50,100 \mu \mathrm{M})$ of propofol or its solvent, $0.1 \%$ dimethyl sulfoxide (DMSO), and exposed to hypoxic or inflammation condition. We intended to identify the effect of hypoxia, inflammation and propofol on the production of BDNF and the expression/truncation/phosphorylation of TrkB in hippocampal neurons and astrocytes. More importantly, we aimed to investigate the underlying mechanisms, including extracellular regulated protein kinase (ERK), cAMP-response element binding protein (CREB), p35 and cyclin-dependent kinase 5 (Cdk5). To confirm the role of these factors, specific inhibitors and short interference RNAs (siRNAs) were applied.

\section{Cell culture}

Primary rat hippocampal neurons and astrocytes were purchased from ScienCell Research Laboratories (Carlsbad, CA, USA). The cryopreserved primary rat hippocampal neurons were thawed and seeded into tissue culture flasks containing $5 \mathrm{ml}$ Neuronal Medium, which was supplemented with Neuronal Growth 
Supplement and $1 \%$ penicillin/streptomycin. The culture media was replaced every 2-3 days. Neurons were incubated at $37^{\circ} \mathrm{C}$ in a humidified atmosphere with $5 \% \mathrm{CO}_{2}$, and were ready for experiments without sub-culture.

The cryopreserved rat astrocytes were thawed and seeded into tissue culture flasks containing $5 \mathrm{ml}$ Astrocyte Medium-animal, which was supplemented with $2 \%$ fetal bovine serum (FBS), Astrocyte Growth Supplement-animal and $1 \%$ penicillin/streptomycin. Astrocytes were incubated at $37^{\circ} \mathrm{C}$ in a humidified atmosphere with $5 \% \mathrm{CO}_{2}$, and culture media was replaced every 2-3 days. The cells were sub-cultured when reaching $80-90 \%$ confluency, and the $4^{\text {th }}$ passage of astrocytes was used in the present study.

\section{Protein preparation and measurement by Western blot analysis}

For total cellular protein isolation, hippocampal neurons and astrocytes were washed with phosphate buffer saline (PBS) and scraped off the culture flasks. After centrifugation for $5 \mathrm{~min}$ at 1000 revolutions per minute (rpm), cell pellets were suspended in RIPA lysis buffer containing $1 \%$ protease inhibitor and $0.1 \%$ phosphatase inhibitor for $5 \mathrm{~min}$, followed by vortexing for $1 \mathrm{~min}$. The proteins were obtained by centrifuging for $5 \mathrm{~min}$ at $3000 \mathrm{rpm}$, and total cellular protein was quantified by BCA assay kit (Beyotime Institute of Biotechnology, Shanghai, China).

Equal amounts of protein (about $60 \mu \mathrm{g}$ ) were separated via $8 \%$ or $10 \%$ SDS-PAGE and electrophoretically transferred to polyvinylidinene fluoride membranes (Millipore Sigma, Shanghai, China). Following blocking in $5 \%$ skimmed milk at room temperature for $2 \mathrm{~h}$, the membranes were incubated overnight at $4^{\circ} \mathrm{C}$ with the following primary antibodies purchased from Cell Signaling Technology (MA, USA): anti-

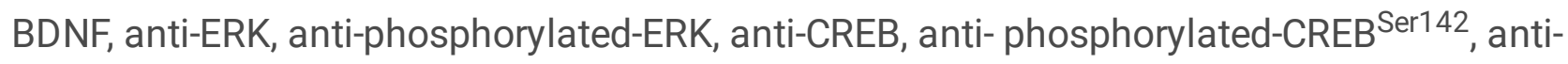

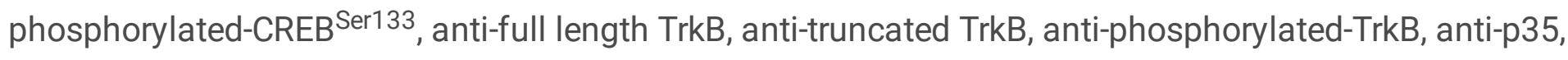
anti-p39,anti-Cdk5 and anti-GAPDH. Subsequently, the membranes were washed and incubated with corresponding HRP-conjugated secondary antibody (Santa Cruz Biotechnology, CA, USA) at room temperature for $2 \mathrm{~h}$. Protein bands were visualized with Amersham ECL plus Western blotting detection reagent (Santa Cruz Biotechnology, CA, USA), and semi-quantified with Image J v1.8.0 software.

\section{Transient transfection of SiRNA}

In this in vitro study, we used siRNA technology to knock down specific gene expression. Cdk5 siRNA (sc29263), p35 siRNA (sc-36154), p39 siRNA (sc-42157) and control siRNA (sc-37007) were purchased from Santa Cruz Biotechnology (CA, USA). siRNAs were delivered to hippocampal neurons using siRNA transfection reagent (sc-29528, Santa Cruz Biotechnology, CA, USA) according to the manufacturer's instructions. Briefly, for each transfection, dilute $5 \mu \mathrm{l}$ siRNA duplex (50 pmol siRNA) into $100 \mu \mathrm{l}$ siRNA transfection medium to obtain solution $A$, and dilute $5 \mu$ l siRNA transfection reagent into $100 \mu \mathrm{l}$ siRNA transfection medium to obtain solution B. Mix solution A and solution B gently and incubate the transfection reagent mixture for $30 \mathrm{~min}$ at room temperature. Hippocampal neurons were seeded in a 6well tissue culture plate and cultured till reaching about $60-70 \%$ confluence. Wash the neurons with siRNA 
transfection medium, overlay $0.5 \mathrm{ml}$ transfection mixture onto the washed neurons, and incubate the neurons for $6 \mathrm{~h}$ in $37^{\circ} \mathrm{C}$ incubator. Then, remove the transfection mixture, replace with normal growth medium, and incubate the neurons for an additional 18 hours in $37^{\circ} \mathrm{C}$ incubator. Thereafter, neurons are ready for experiments.

\section{Measurement of Cdk5 kinase activity}

Cdk5 kinase activity was analyzed by fluorescence assay using commercially available ELISA kits (Weike Biological Technology Company, Shanghai, China) according to the manufacturer's instructions.

In brief, hippocampal neurons were seeded in 96-well plates and subject to respective treatment. Then, neurons were washed and incubated with $200 \mu \mathrm{L}$ Cdk5 kinase substrates solution supplemented with $5 \mathrm{mM} \mathrm{MgCl} 2$ and $0.5 \mathrm{mM} \mathrm{ATP}$ for $2 \mathrm{~h}$ at $37^{\circ} \mathrm{C}$. Cell culture plates were subject to a Clariostar TM spectrofluorimeter, and fluorescence emission was recorded at $680 \mathrm{~nm}$ following excitation at $620 \mathrm{~nm}$. Cdk5 kinase fluorescence was calculated by substraction of fluorescence from the values obtained in the absence of Cdk5 kinase. Experiments were performed in triplicate, and data were expressed as percentage of relative fluorescence compared with that of untreated control neurons.

\section{Statistical analysis}

Data were presented as mean \pm standard deviation. All experiments were conducted with five independent repeats, which were performed with different cultures. Differences between groups were assessed with paired, two-tailed Student's t-test or one-way ANOVA, followed by post hoc Tukey testing. All statistical analyses were performed with SPSS software 11.5, and a significant difference was set at $p<0.05$.

\section{Results}

Hypoxia and TNF-a reduced BDNF production in rat hippocampal neurons and astrocytes.

Rat hippocampal neurons and astrocytes were cultured and exposed to hypoxia $\left(5 \% \mathrm{O}_{2}\right)$ or TNF-a $(40 \mathrm{ng} / \mathrm{mL})$ treatment for different times $(0,1,2,3,6,12 \mathrm{~h})$, and the production of BDNF was measured. As shown in Figure 1, we reported that in hippocampal neurons and astrocytes, hypoxia reduced BDNF production in a time-dependent manner, with the significant effects appearing at $3 \mathrm{~h}$ in hippocampal neurons (Figure $1 a, p<0.01$ vs control) and at $6 \mathrm{~h}$ in astrocytes (Figure $1 \mathrm{~b}, \mathrm{p}<0.01$ vs control). In addition, we found that TNF- $a$ also reduced BDNF production in a time-dependent manner, and the significant effects appeared at $3 \mathrm{~h}$ in both hippocampal neurons and astrocytes (Figure $1 \mathrm{c}$ and $1 \mathrm{~d}, \mathrm{p}<0.01 \mathrm{vs}$ control). Thereafter, these treatment conditions were applied in the following experiments to study the potential mechanisms.

Propofol reversed hypoxia- and TNF-a-modulated BDNF reduction in rat hippocampal neurons 
To observe the effects of propofol on hypoxia- and TNF-a- modulated BDNF reduction in hippocampal neurons and astrocytes, we pretreated cells with different concentrations of propofol $(1,5,10,25,50$, $100 \mu \mathrm{M}$ ) for $1 \mathrm{~h}$, followed by hypoxia or TNF-a treatment. As shown in Figure 2, in hippocampal neurons, propofol $(25,50$ and $100 \mu \mathrm{M})$ induced BDNF production, which was inhibited by hypoxia $\left(5 \% \mathrm{O}_{2}, 3 \mathrm{~h}\right)$ treatment (Figure $2 a, p<0.01 v s$ control, $p<0.05$ vs hypoxia). Propofol $(25,50$ and $100 \mu \mathrm{M})$ also induced BDNF production, which was inhibited by TNF-a $(40 \mathrm{ng} / \mathrm{mL}, 3 \mathrm{~h})$ treatment (Figure $2 b, p<0.01$ vs control, $\mathrm{p}<0.05$ vs TNF-a). In contrast, we found that even $100 \mu \mathrm{M}$ propofol had no or minor effect on BDNF production in astrocytes in response to hypoxia or TNF-a (Figure $2 \mathrm{c}$ and $2 \mathrm{~d}$ ). Also, please note that $0.1 \%$ DMSO, the solvent for propofol, had no effect on BDNF production in hippocampal neurons or astrocytes (Figure 2). Therefore, we ruled out the role of DMSO. More importantly, we inferred that $25 \mu \mathrm{M}$ propofol might be the minimally effective concentration that reversed hypoxia- and TNF-a-inhibited BDNF production in hippocampal neurons, and accordingly we focused on the mechanism responsible for the beneficial effect of $25 \mu \mathrm{M}$ propofol.

The beneficial effect of popofol on BDNF production was mediated through regulating the phosphorylation of ERK and CREB

We revealed that in rat hippocampal neurons, hypoxia $\left(5 \% \mathrm{O}_{2}, 3 \mathrm{~h}\right)$ and TNF- $\mathrm{a}(40 \mathrm{ng} / \mathrm{mL}, 3 \mathrm{~h})$ increased

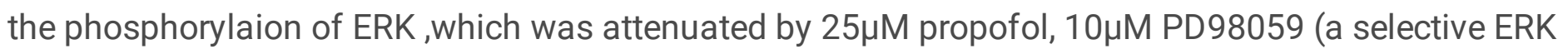
inhibitor) or 10 $1 \mathrm{M} \mathrm{KO}-947$ (a potent and specific ERK inhibitor) (Figure 3a). We also detected that hypoxia $\left(5 \% \mathrm{O}_{2}, 3 \mathrm{~h}\right)$ and TNF-a $(40 \mathrm{ng} / \mathrm{mL}, 3 \mathrm{~h})$ increased the phosphorylation of CREB at Ser142 (p-CREB ${ }^{\text {Ser142) }}$ while reduced the phosphorylation of CREB at Ser133 ( $p$-CREB ${ }^{\operatorname{Ser} 133}$ ), which were both reversed by $25 \mu \mathrm{M}$

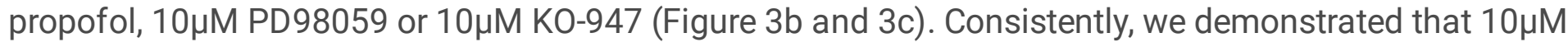
PD98059 and 10 $1 \mathrm{M}$ KO-947 could attenuate the inhibitory effect of hypoxia and TNF-a on BDNF production, which is similar to the effect of propofol (Figure 3d). In addition, we reported that the beneficial effect of propofol on hypoxia- and TNF-a-inhibited BDNF production was abolished by the

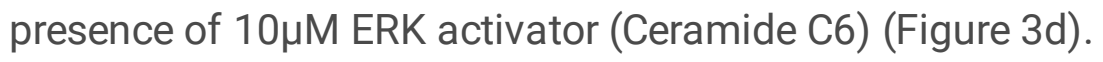

Hypoxia and TNF-a had no effect on TrkB expression, truncation or phosphorylation in rat hippocampal neurons and astrocytes.

Rat hippocampal neurons and astrocytes were cultured and exposed to hypoxia $\left(5 \% \mathrm{O}_{2}\right)$ or TNF-a $(40 \mathrm{ng} / \mathrm{mL})$ treatment for different times $(0,1,2,3,6,12 \mathrm{~h})$, and the expression, truncation, as well as phosphorylation of TrkB were measured. As shown in Figure 4, we reported that hypoxia had no effect on the expression, truncation or phosphorylation of TrkB in rat hippocampal neurons (Figure 4a) and in astrocytes (Figure 4b). Also, TNF-a had no effect on the expression, truncation or phosphorylation of TrkB in rat hippocampal neurons (Figure 4c) and astrocytes (Figure 4d).

Propofol induced TrkB phosphorylation in rat hippocampal neurons 
We treated rat hippocampal neurons and astrocytes with different concentrations of propofol $(1,5,10,25$, $50,100 \mu \mathrm{M})$ for $1 \mathrm{~h}$, followed by hypoxia $\left(5 \% \mathrm{O}_{2}, 3 \mathrm{~h}\right)$ or TNF-a $(40 \mathrm{ng} / \mathrm{mL}, 3 \mathrm{~h})$ treatment, and examined the expression, truncation and phosphorylation of TrkB. Interestingly, we noticed that in rat hippocampal neurons propofol had no effect on TrkB expression or truncation, while propofol (50 and 100 $\mu \mathrm{M})$ induced TrkB phosphorylation no matter cells were exposed to hypoxia, TNF-a or not (Figure 5a, $p<0.05$ vs control). However propofol had no effect on TrkB expression, truncation or phosphorylation in astrocytes (Figure 5b). Thereafter, we intended to investigate the mechanism responsible for $50 \mu \mathrm{M}$ propofol-induced TrkB phosphorylation in hippocampal neurons.

\section{Propofol-induced TrkB phosphorylation was carried out via modulating p35 expression and} Cdk5activation

We found that in hippocampal neurons, hypoxia $\left(5 \% \mathrm{O}_{2}, 3 \mathrm{~h}\right)$ and TNF-a $(40 \mathrm{ng} / \mathrm{mL}, 3 \mathrm{~h})$ did not affect p35 expression, while, $50 \mu \mathrm{M}$ propofol, rather than $0.1 \% \mathrm{DMSO}$, induced the expression of p35 regardless of the exposure to hypoxia or TNF-a (Figure 6a). Consistently, although hypoxia and TNF-a had no effect on the activation of Cdk5 (Figure $6 \mathrm{~b}$ ), it was activated by $50 \mu \mathrm{M}$ propofol but not $0.1 \% \mathrm{DMSO}$. In addition, hypoxia, TNF-a, propofol and DMSO had no effect on the expression of Cdk5 and p39 (Figure 6c). Then, we applied siRNA technology to confirm the involvement of p35 and Cdk5 in propofol-mediated TrkB phosphorylation. As shown in Figure 6d, we demonstrated that the siRNA targeting p35, p39 and Cdk5 could effectively diminish the expression of p35, p39 and Cdk5, respectively. More importantly, we revealed that blockade of p35 and Cdk5 alleviated propofol-induced TrkB phosphorylation, while blockade of p39 had no such effect (Figure 6e).

\section{Discussion}

\section{Hypoxia- and TNF-a-mediated dysregulation of BDNF/TrkB pathway}

BDNF belongs to the neurotrophin (NT) family, which is composed of four structurally related members: BDNF, neuronal growth factor (NGF), neurotrophin-3 (NT-3) and NT-4/5 [25]. It has been well recognized that BDNF is the most abundant endogenous neurotrophic factor in the body, and reduced levels of BDNF were reported to play a key role in rodent models during the development of neurological disorders, such as cerebral ischemia-reperfusion injury [2] and neuroinflammation-related brain injury [3]. Besides, it is clear that the NT actions are mediated by interacting with two transmembrane receptors with different affinity. Generally, all members of the NT family bind to p75NTR with low affinity, whereas mature NTs bind to different Trk receptors, including TrkA, TrkB and TrkC, with high affinity according to ligand selectivity. TrkA has been identified as the preferred receptor for NGF, and TrkB for BDNF, and TrkC for NT$3 / 4 / 5$ [26]. After bound by BDNF, TrkB undergoes dimerization, followed by phosphorylation of intracellular tyrosine kinase residues, and acts as docking sites for adaptor proteins that allow additional kinases to be recruited for activation of intracellular signaling pathways. The activation of BDNF/TrkB is required for neuron differentiation, survival, synaptic plasticity and neurotransmitter regulation, while 
dysregulation of BDNF/TrkB contributes to many pathological processes, including traumatic brain injury, brain ischemic injury, and neurodegenerative diseases [13].

It is known that BDNF/TrkB dysregulation was correlated with several vicious factors, such as oxidative stress and inflammation [27]. In the current study we focused on two factors (hypoxia and inflammation) which are major stimuli during the development of neurological disorders, and two cell types (hippocampal neurons and astrocytes) which are major sources of BDNF in CNS. We found that both hypoxia and inflammation reduced the expression of BDNF in hippocampal neurons and astrocytes (Figure 1). However, they had no effect on TrkB expression囚truncation or phosphorylation (Figure 4). Since we only focused the role of mature BDNF in this study, we did not examine p75NTR and TrkA as well as TrkC. In addition, it is known that TrkB has two isoforms: truncated TrkB (TrkB-TC) and full length TrkB (TrkB-FL). TrkB-TC may act as negative modulators of TrkB-FL. A previous study showed that excitotoxic stimulation of cultured rat hippocampal neurons with glutamate downregulated TrkB-FL while upregulated TrkB-TC, which resulted in dysregulation of BDNF/TrkB signaling [28]. Nevertheless, we found neither hypoxia nor TNF-a affected the truncation of TrkB (Figure 4). Interestingly, our findings are inconsistent with a previous animal study that reported chronic cerebral ischemia may increase BDNF and TrkB expression in the hippocampus of aged rats [24]. We postulated that the discrepancy could be due to two reasons: firstly, we examined acute hypoxia and inflammation rather than chronic ischemia; secondly, our study was carried out in neurons rather than in aged animals. Anyway, we concluded that in hippocampal neurons and astrocytes, hypoxia and inflammation may cause dysregulation of BDNF/TrkB pathway mainly through affecting BDNF expression.

The protective property of propofol against hypoxia- and TNF-a-mediated of BDNF/TrkB dysregulation

Propofol is an intravenous anesthetic widely used in clinical anesthesia and sedation. In addition, it has a variety of biological effects on organ protection, including brain [29], heart [30] and kidney [31]. Nowadays, the neuro-protective property of propofol in the CNS and the underlying mechanism are of great interests. A large amount of in vitro studies revealed that propofol may improve BBB function [21], protect neuron apoptosis [18] and autophagy [17], and maintain microglia function [32]. In addition, animal studies demonstrated that propofol may improve brain function in rats with ischemia-reperfusion injury [33] and may ameliorate neuroinflammatory injury in rats [34, 35].

Recently, the role of BDNF/TrkB signaling in the neuro-protective property of propofol gains interests. An animal study indicated that propofol may protect chronic ischemic cerebral injury in aged rats via modulating BDNF/TrkB pathway [24]. In that animal study, it was reported that low-dose of propofol (10 $\mathrm{mg} / \mathrm{kg}$, intraperitoneally) promoted the expression of BDNF and TrkB, but high-dose of propofol (50 $\mathrm{mg} / \mathrm{kg}$, intraperitoneally) inhibited their expression. Consistently, our in vitro study demonstrated that 25$50 \mu \mathrm{M}$ propofol induced BDNF expression in hippocampal neurons which are exposed to hypoxia and TNF-a (Figure 2). Meanwhile, we found propofol had no effect on TrkB expression, while increased its phosphorylation no matter hippocampal neurons were exposed to hypoxia/TNF-a or not (Figure 5). We postulated that the difference in the amount of propofol administration and the difference in experiment 
model may account for the discrepancy. In contrast, our data implied that astrocytes may not be a target for propofol in regarding to BDNF/TrkB dysregulation (Figure 2 and 5). It is noted that in our study, the beneficial concentration of propofol was $25-50 \mu \mathrm{M}$, which is within the plasma range of propofol during general anesthesia and is clinically relevant. Accordingly, we concluded that propofol may regulate hypoxia- and TNF-a-mediated BDNF/TrkB dysregulation, through both affecting BDNF expression and affecting TrkB phosphorylation only in hippocampal neurons.

ERK/CREB and p35/Cdk5 were involved in the beneficial effect of propofol against hypoxia- and TNF-amediated BDNF/TrkB dysregulation

The mechanism involved in the neuro-protective effect of propofol against hypoxia- and inflammationmediated injuries has been widely studied both in the in vitro model and in the animal model, and may include but not be limited to phosphatidylinositol-3-kinase/protein kinase B (PI3K/PKB) pathway [34], PIM-1/nitric oxide synthase (NOS)/NO pathway [36], rapamycin/ribosomal protein S6 kinase beta-1 pathway [37], janus kinase/signal transducer and activator of transcription (JAK/STAT) pathway [38], HSF1/heat shock protein 27 (HSP27) and Nrf2/ HSP32 pathway [22], and $\mathrm{Ca}^{2+} /$ calmodulin-dependent protein kinase II (CAMKII)/extracellular regulated protein kinases (ERK)/NF-KB pathway [21, 23]. However, the molecular mechanism responsible for propofol-modulated BDNF/TrkB regulation still remains unknown.

Here is the present study, our data suggested that ERK/CREB is involved in hypoxia-and TNF-a-mediated BDNF/TrkB dysregulation (Figure 3), and more importantly, we believed that ERK/CREB plays a key role in the beneficial effect of propofol on BDNF production, because the presence of ERK activator markedly abolished the beneficial effects of propofol on BDNF production (Figure 3). The pivotal role of ERK/CREB in BDNF production has previously been proved in the brain of mice [39] and rats [40]. It is well-known that CREB could be phosphorylated by protein kinases such as protein kinase $A$ (PKA), protein kinase $C$ (PKC), PI3K, CAMKII and ERK at different site such as Ser133 and Ser142, and it is recognized that most kinases induce $\mathrm{p}-\mathrm{CREB}^{\mathrm{Ser} 133}$, which increases CREB transcriptional activity, while some kinases induce p-

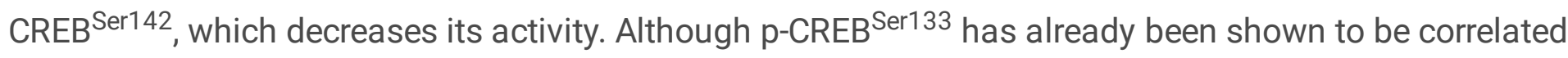
with BDNF production in rat model [41] and in rat cortical neurons [42] as well as in mouse hippocampal neurons [43], the role of $p-\mathrm{CREB}^{\mathrm{Ser} 142}$ has rarely been investigated. One of the novelties of this study is that we examined $\mathrm{p}$-CREB ${ }^{\mathrm{Ser} 142}$, and we found that propofol-induced BDNF production was mediated through increasing $\mathrm{p}-\mathrm{CREB}^{\mathrm{Ser} 133}$ and decreasing $\mathrm{p}-\mathrm{CREB}^{\mathrm{Ser} 142}$ simultaneously.

In addition, our data implied that p35/Cdk5 is involved in hypoxia- and TNF-a-mediated BDNF/TrkB dysregulation (Figure 5), and our findings clearly indicated that p35/Cdk5 is responsible for the beneficial effect of propofol on TrkB phosphorylation, because the blockade of p35/Cdk5 expression almost completely abolished the beneficial effects of propofol on TrkB phosphorylation (Figure 5). Cdk5 is a small serine/threonine kinase abundant in postmitotic neurons, and the activation of Cdk5 requires the binding of one of its two specific activators, p35 or p39, in the developing cerebral cortex and hippocampus [44, 45]. It is known p35 and p39 share approximately $60 \%$ sequence homology and exhibit 
differential developmental expression in the brain. The expression of p35 protein is high throughout the embryonic stage, whereas that of p39 increases during postnatal differentiation. Although in vitro experiments suggest that p35 and p39 share similar substrate specificity, they are spatially segregated within neurons and have different biochemical properties [46]. Previous study indicated that p35/Cdk5mediated phosphorylation of target protein is required for hypoxia-induced xanthine oxidoreductase hyperactivation in the lung [47], and p35/Cdk5 has been proved to be responsible for phosphorylation of TrkB, neurofilament proteins and tau protein in the brain $[45,48]$. Consistently, we found p35, rather than p39 is critical for Cdk5 activation and TrkB phosphorylation in the hippocampal neurons that were exposed to hypoxia, TNF-a and propofol (Figure 5).

\section{Limitations}

We realized that there are several limitations within this study. Firstly, we only detected that ERK/CREB and $\mathrm{p} 35 / \mathrm{Cdk} 5$ were involved in hypoxia- and TNF-a-as well as propofol -mediated regulation of BDNF/TrkB pathway, no detailed signaling pathway was further investigated. Actually, we are working on this issue, trying to reveal how these factors modulate ERK phosphorylation and p35 expression. Secondly, it is known that p-CREB may be dephosphorylated by phosphotase PP1 and PP2A to keep the balance of its phosphorylation status. However, in the study, we did not examine the effect of hypoxia, TNF-a- or propofol on the expression and activity of these enzymes.

\section{Conclusion}

In this in vitro study, we reported that in rat hippocampal neurons propofol, via modulating ERK/CREB signaling pathway, may reverse hypoxia- and TNF-a-mediated reduction of BDNF. In addition, we demonstrated that in hippocampal neurons propofol, via activting $\mathrm{p} 35 / \mathrm{Cdk} 5$ pathway, may induce TrkB phosphorylation. Taken together, our findings suggested a novel protective effect and mechanism of propofol against hypoxia- and TNF-a-induced malfunction of hippocamal neurons.

\section{Declarations}

Funding: Not applicable.

Conflicts of interest/Competing interests: The authors declare that they have no conflict of interest or competing interest.

Availability of data and material: All data generated or analyzed during this study are included in this manuscript. 
Code availability: Not applicable.

Authors' contributions: Weiping Tao performed research and wrote the paper; Xuesong Zhang performed research and wrote the paper; Juan Ding performed research and analyzed data; Shijian Yu performed research; Peiqing Ge analyzed data; Jingfeng Han analyzed data; Xing Luo analyzed data and wrote the paper; Wei Cui designed research and revised the paper; Jiawei Chen designed research and revised the paper.

\section{Ethics approval and consent to participate]}

Not applicable.

\section{Consent for publication!}

All authors have read and approved the manuscript, and agreed to its submission as well as publication in "Molecular Neurobiology".

\section{Acknowledgements $\square$}

Not applicable.

\section{References}

1. Chen SD, Wu CL, Hwang WC, Yang DI (2009) More insight into BDNF against neurodegeneration: anti-apoptosis, anti-oxidation, and suppression of autophagy. Int J Mol Sci 18(3):545

2. Wang L, Jiang J, Zhou T, Xue X, Cao Y (2021) Improvement of cerebral ischemia-reperfusion injury via regulation of apoptosis by exosomes derived from BDNF-overexpressing HEK293. Biomed Res Int 2021:6613510

3. Lima GB, Doorduin J, Klein HC, Dierckx RAJO, Bromberg E, de Vries EFJ (2019) Brain-derived neurotrophic factor in brain disorders: focus on neuroinflammation. Mol Neurobiol 56(5):3295-3312

4. Mizoguchi Y, Yao H, Imamura Y, Hashimoto M, Monji A (2020) Lower brain-derived neurotrophic factor levels are associated with age-related memory impairment in community-dwelling older adults: the Sefuri study. Sci Rep 10(1):16442

5. Chen H, Cao J, Zha L, Wang P, Liu Z, Guo B, Zhang G, Sun Y, Zhang Z, Wang Y (2020) Neuroprotective and neurogenic effects of novel tetramethylpyrazine derivative T-006 in Parkinson's disease models through activating the MEF2-PGC1alpha and BDNF/CREB pathways Aging (Albany NY) 12(14):14897-14917

6. Choung JS, Kim JM, Ko MH, Cho DS, Kim M (2021) Therapeutic efficacy of repetitive transcranial magnetic stimulation in an animal model of Alzheimer's disease. Sci Rep 11(1):437

7. Jiang Y, Gao H, Yuan H, Xu H, Tian M, Du G, Xie W (2019) Amelioration of postoperative cognitive dysfunction in mice by mesenchymal stem cell-conditioned medium treatments is associated with 
reduced inflammation, oxidative stress and increased BDNF expression in brain tissues. Neurosci Lett 709:134372

8. Chao MV, Hempstead BL (1995) p75 and Trk: a two-receptor system. Trends Neurosci 18(7):321-326

9. Colucci-D'Amato L, Speranza L, Volpicelli F (2020) Neurotrophic factor BDNF, physiological functions and therapeutic potential in depression, neurodegeneration and brain cancer. Int $\mathrm{J} \mathrm{Mol} \mathrm{Sci}$ 21(20):7777

10. Zhang JC, Yao W, Hashimoto K (2016) Brain-derived neurotrophic factor (BDNF)-TrkB signaling in inflammation-related depression and potential therapeutic targets. Curr Neuropharmacol 14(7):721731

11. He Y, Chen S, Tsoi B, Qi S, Gu B, Wang Z, Peng C, Shen J (2021) Alpinia oxyphylla miq and its active compound P-coumaric acid promote brain-derived neurotrophic factor signaling for inducing hippocampal neurogenesis and improving post-cerebral ischemic spatial cognitive functions. Front Cell Dev Biol 8:577790

12. Wang W, Zhang Y, Yu W, Gao W, Shen N, Jin B, Wang X, Fang C, Wang Y (2020) Bushenhuoxue improves cognitive function and activates brain-derived neurotrophic factor-mediated signaling in a rat model of vascular dementia. J Tradit Chin Med 40(1):49-58

13. Qiu LL, Pan W, Luo D, Zhang GF, Zhou ZQ, Sun XY, Yang JJ, Ji MH (2020) Dysregulation of $\mathrm{BDNF} / \mathrm{TrkB}$ signaling mediated by NMDAR/Ca ${ }^{2+} /$ calpain might contribute to postoperative cognitive dysfunction in aging mice. J Neuroinflammation 17(1):23

14. Alhayyan A, McSorley S, Roxburgh C, Kearns R, Horgan P, McMillan D (2019) The effect of anesthesia on the postoperative systemic inflammatory response in patients undergoing surgery: $A$ systematic review and meta-analysis. Surg Open Sci 2(1):1-21

15. Leurcharusmee P, Sawaddiruk P, Punjasawadwong Y, Chattipakorn N, Chattipakorn SC (2018) The Possible Pathophysiological Outcomes and Mechanisms of Tourniquet-Induced IschemiaReperfusion Injury during Total Knee Arthroplasty. Oxid Med Cell Longev 2018:8087598

16. Fan W, Zhu X, Wu L, Wu Z, Li D, Huang F, He H (2015) Propofol: an anesthetic possessing neuroprotective effects. Eur Rev Med Pharmacol Sci 19(8):1520-1529

17. Li Y, He Z, Lv H, Chen W, Chen J (2020) Calpain-2 plays a pivotal role in the inhibitory effects of propofol against TNF-alpha-induced autophagy in mouse hippocampal neurons. J Cell Mol Med 24(16):9287-9299

18. Xu Z, Lu Y, Wang J, Ding X, Chen J, Miao C (2017) The protective effect of propofol against TNF-ainduced apoptosis was mediated via inhibiting iNOS/NO production and maintaining intracellular Ca2 + homeostasis in mouse hippocampal HT22 cells. Biomed Pharmacother 91:664-672

19. Lu Y, Chen W, Lin C, Wang J, Zhu M, Chen J, Miao C (2017) The protective effects of propofol against $\mathrm{CoCl}_{2}$-induced $\mathrm{HT} 22$ cell hypoxia injury via PP2A/CAMKIla/nNOS pathway. BMC Anesthesiol 17:32

20. Chen J, Chen W, Zhu M, Zhu Y, Xu P, Miao C (2015) Angiotensin Il-induced mouse hippocampal neuronal HT22 cell apoptosis was inhibited by propofol: Role of neuronal nitric oxide synthase and metallothinonein-3. Neuroscience 305:117-127 
21. Chen W, Ju XZ, Lu Y, Ding XW, Miao CH, Chen JW (2019) Propofol improved hypoxia-impaired integrity of blood-brain barrier via modulating the expression and phosphorylation of zonula occludens-1. CNS Neurosci Ther 25(6):704-713

22. Sun X, Yin Y, Kong L, Chen W, Miao C, Chen J (2019) The effect of propofol on hypoxia-modulated expression of heat shock proteins: potential mechanism in modulating blood-brain barrier permeability. Mol Cell Biochem 462(1-2):85-96

23. Ding XW, Sun X, Shen XF, Lu Y, Wang JQ, Sun ZR, Miao CH, Chen JW (2019) Propofol attenuates TNF-a-induced MMP-9 expression in human cerebral microvascular endothelial cells by inhibiting Ca2+/CAMK II/ERK/NF-KB signaling pathway. Acta Pharmacol Sin 40(10):1303-1313

24. Chen G, Fu Q, Cao JB, Mi WD (2012) Effect of propofol on brain-derived neurotrophic factor and tyrosine kinase receptor $B$ in the hippocampus of aged rats with chronic cerebral ischemia. Neural Regen Res 7(21):1645-1649

25. Chao MV, Rajagopal R, Lee FS (2006) Neurotrophin signalling in health and disease. Clin Sci 110:167-173

26. László A, Lénárt L, Illésy L, Fekete A, Nemcsik J (2019) The role of neurotrophins in psychopathology and cardiovascular diseases: Psychosomatic connections. J Neural Transm 126:265-278

27. Hao Y, Xiong R, Gong X (2021) Memantine, NMDA Receptor Antagonist, Attenuates ox-LDL-Induced Inflammation and Oxidative Stress via Activation of BDNF/TrkB Signaling Pathway in HUVECs. Inflammation 44(2):659-670

28. Gomes JR, Costa JT, Melo CV, Felizzi F, Monteiro P, Pinto MJ, Inacio AR, Wieloch T, Almeida RD, Graos M, Duarte CB (2012) Excitotoxicity downregulates TrkB.FL signaling and upregulates the neuroprotective truncated TrkB receptors in cultured hippocampal and striatal neurons. J Neurosci 32:4610-4622

29. Jia L, Wang F, Gu X, Weng Y, Sheng M, Wang G, Li S, Du H, Yu W (2017) Propofol postconditioning attenuates hippocampus ischemia-reperfusion injury via modulating JAK2/STAT3 pathway in rats after autogenous orthotropic liver transplantation. Brain Res 1657:202-207

30. Zhu A, Wei X, Zhang Y, You T, Yao S, Yuan S, Xu H, Li F, Mao W (2017) Propofol Provides Cardiac Protection by Suppressing the Proteasome Degradation of Caveolin-3 in Ischemic/Reperfused Rat Hearts. J Cardiovasc Pharmacol 69(3):170-177

31. Wei Q, Zhao J, Zhou X, Yu L, Liu Z, Chang Y (2019) Propofol can suppress renal ischemia-reperfusion injury through the activation of PI3K/AKT/mTOR signal pathway. Gene 708:14-20

32. Lu Y, Gu Y, Ding X, Wang J, Chen J, Miao C (2017) Intracellular Ca2 + homeostasis and JAK1/STAT3 pathway are involved in the protective effect of propofol on BV2 microglia against hypoxia-induced inflammation and apoptosis. PLoS One 12.e0178098

33. Chen Y, Li Z (2021) Protective Effects of Propofol on Rats with Cerebral Ischemia-Reperfusion Injury Via the PI3K/Akt Pathway. J Mol Neurosci 71(4):810-820

34. Ma Z, Li K, Chen P, Pan J, Li X, Zhao G (2020) Propofol Attenuates Inflammatory Damage via Inhibiting NLRP1-Casp1-Casp6 Signaling in Ischemic Brain Injury. Biol Pharm Bull 43(10):1481-1489 
35. Jiang P, Jiang Q, Yan Y, Hou Z, Luo D (2021) Propofol ameliorates neuropathic pain and neuroinflammation through PPAR gamma up-regulation to block Wnt/beta-catenin pathway. Neurol Res 43(1):71-77

36. Yu Y, Xu Z, Shen F, Lin R, Li H, Lv X, Liu Z (2020) Propofol Protects Against TNF-alpha-induced Bloodbrain Barrier Disruption via the PIM-1/eNOS/NO Pathway. Curr Neurovasc Res 17(4):471-479

37. Wang Y, Tian D, Wei C, Cui V, Wang H, Zhu Y, Wu A, Yue Y (2020) Propofol Attenuates alpha-Synuclein Aggregation and Neuronal Damage in a Mouse Model of Ischemic Stroke. Neurosci Bull 36(3):289298

38. Zhang Y, Zuo Y, Li B, Xie J, Ma Z, Thirupathi A, Yu P, Gao G, Shi M, Zhou C, Xu H, Chang Y, Shi Z (2019) Propofol prevents oxidative stress and apoptosis by regulating iron homeostasis and targeting JAK/STAT3 signaling in SH-SY5Y cells. Brain Res Bull 153:191-201

39. Mi Y, Qi G, Fan R, Qiao Q, Sun Y, Gao Y, Liu X (2017) EGCG ameliorates high-fat- and high-fructoseinduced cognitive defects by regulating the IRS/AKT and ERK/CREB/BDNF signaling pathways in the CNS. FASEB J 31(11):4998-5011

40. Lu C, Wang Y, Xu T, Li Q, Wang D, Zhang L, Fan B, Wang F, Liu X (2018) Genistein Ameliorates Scopolamine-Induced Amnesia in Mice Through the Regulation of the Cholinergic Neurotransmission, Antioxidant System and the ERK/CREB/BDNF Signaling. Front Pharmacol 9:1153

41. Guo C, Liu Y, Fang MS, Li Y, Li W, Mahaman YAR, Zeng K, Xia Y, Ke D, Liu R, Wang JZ, Shen H, Shu X, Wang X (2020) omega-3PUFAs Improve Cognitive Impairments Through Ser133 Phosphorylation of CREB Upregulating BDNF/TrkB Signal in Schizophrenia. Neurotherapeutics 17(3):1271-1286

42. Jeon SJ, Rhee SY, Seo JE, Bak HR, Lee SH, Ryu JH, Cheong JH, Shin CY, Kim GH, Lee YS, Ko KH (2011) Oroxylin A increases BDNF production by activation of MAPK-CREB pathway in rat primary cortical neuronal culture. Neurosci Res 69(3):214-222

43. Lee YS, Park SY, Heo HJ, Lee WS, Hong KW, Kim CD (2019) Multitarget-directed cotreatment with cilostazol and aripiprazole for augmented neuroprotection against oxidative stress-induced toxicity in HT22 mouse hippocampal cells. Eur J Pharmacol 857:172454

44. Tsai LH, Delalle I, Caviness VS, Chae T, Harlow E (1994) p35 is a neural-specific regulatory subunit of cyclin-dependent kinase 5. Nature 371:419-423

45. Lew J, Huang QQ, Qi Z, Winkfein RJ, Aebersold R, Hunt T, Wang JH (1994) A brain-specific activator of cyclin-dependent kinase 5. Nature 371(6496):423-426

46. Asada A, Yamamoto N, Gohda M, Saito T, Hayashi N, Hisanaga S (2008) Myristoylation of p39 and p35 is a determinant of cytoplasmic or nuclear localization of active cyclin-dependent kinase 5 complexes. J Neurochem 106:1325-1336

47. Kim BS, Serebreni L, Fallica J, Hamdan O, Wang L, Johnston L, Kolb T, Damarla M, Damico R, Hassoun PM (2015) Cyclin-dependent kinase five mediates activation of lung xanthine oxidoreductase in response to hypoxia. PLoS One 10(4):e0124189 
48. Lai KO, Wong AS, Cheung MC, Xu P, Liang Z, Lok KC, Xie H, Palko ME, Yung WH, Tessarollo L, Cheung $\mathrm{ZH}$, Ip NY (2012) TrkB phosphorylation by Cdk5 is required for activity-dependent structural plasticity and spatial memory. Nat Neurosci 15(11):1506-1515

\section{Figures}

a

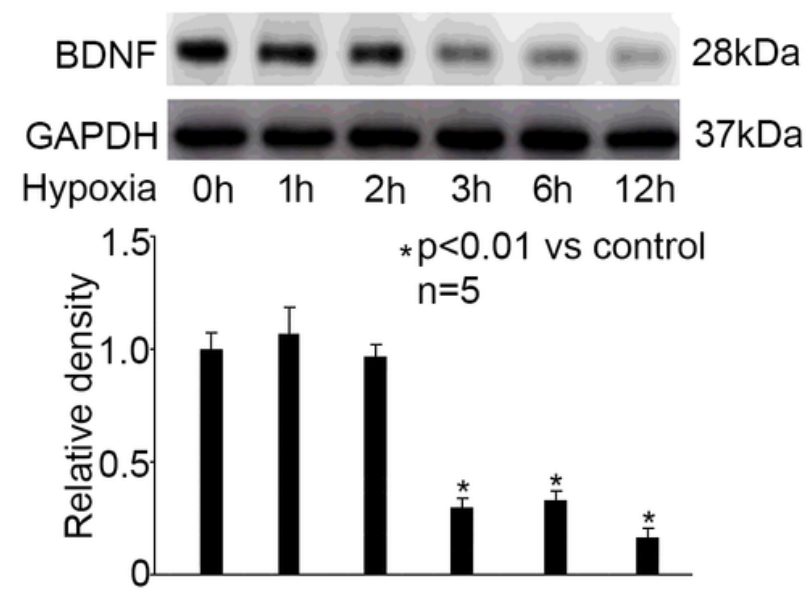

C

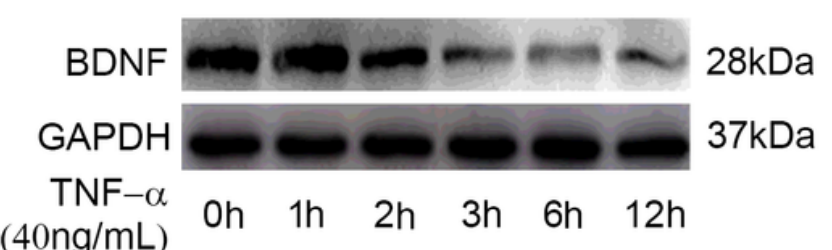

$\mathrm{TN}-\alpha$
$(40 \mathrm{ng} / \mathrm{mL})$ Oh $1 \mathrm{~h} \quad 2 \mathrm{~h} \quad 3 \mathrm{~h} \quad 6 \mathrm{~h} \quad 12 \mathrm{~h}$

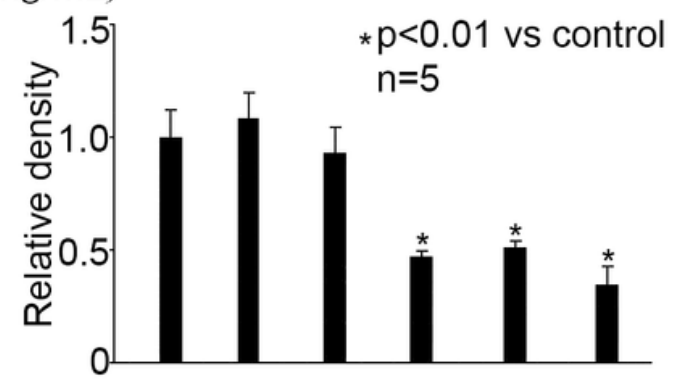

b

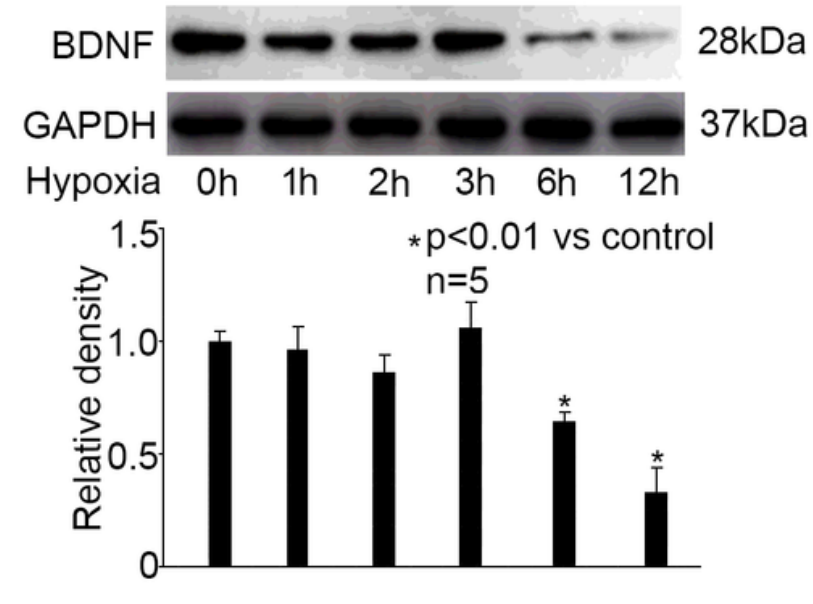

d

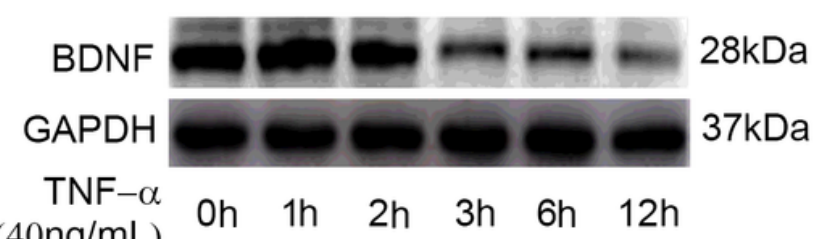

(40ng/mL) $0 \mathrm{~h} \quad 1 \mathrm{~h} \quad 2 \mathrm{~h} \quad 3 \mathrm{~h} \quad 6 \mathrm{~h} \quad 12 \mathrm{~h}$

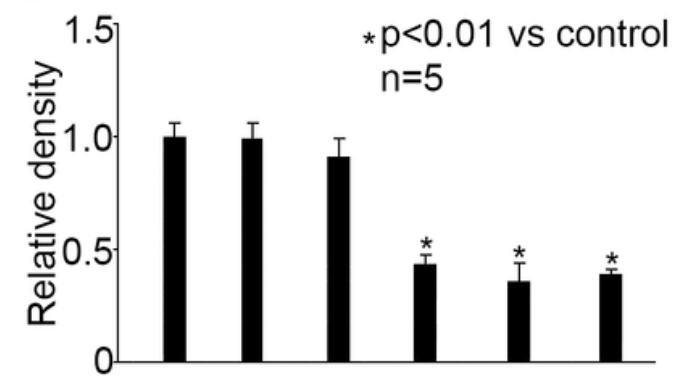

\section{Figure 1}

Hypoxia and TNF-a reduced the production of BDNF in rat hippocampal neurons and astrocytes. The upper panel was a representative experiment and the lower panel was the summary of densitometric data from 5 separate experiments. GAPDH served as loading control. Data were expressed as normalized ratio of protein band density of BDNF against GAPDH, and were presented as mean \pm standard deviation. Hypoxia treatment for $0 \mathrm{~h}$ was considered as normoxic condition and served as control. (a) In 
hippocampal neurons, hypoxia reduced BDNF production in a time-dependent manner. (b) In astrocytes, hypoxia reduced BDNF production in a time-dependent manner. (c) In hippocampal neurons, TNF-a reduced BDNF production in a time-dependent manner. (d) In astrocytes, TNF-a reduced BDNF production in a time-dependent manner.

a

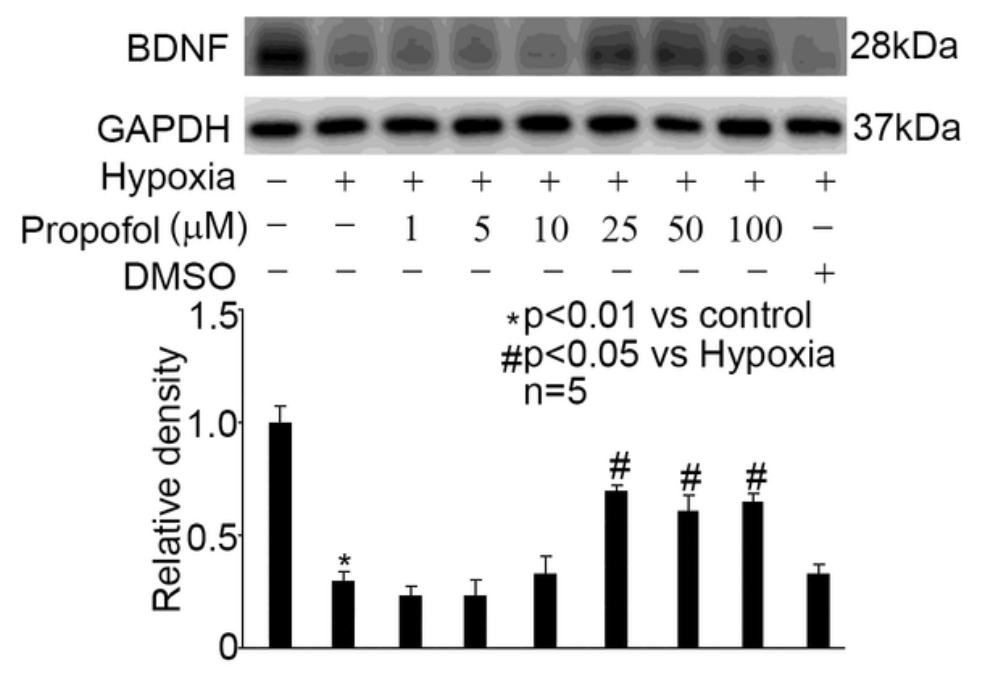

C

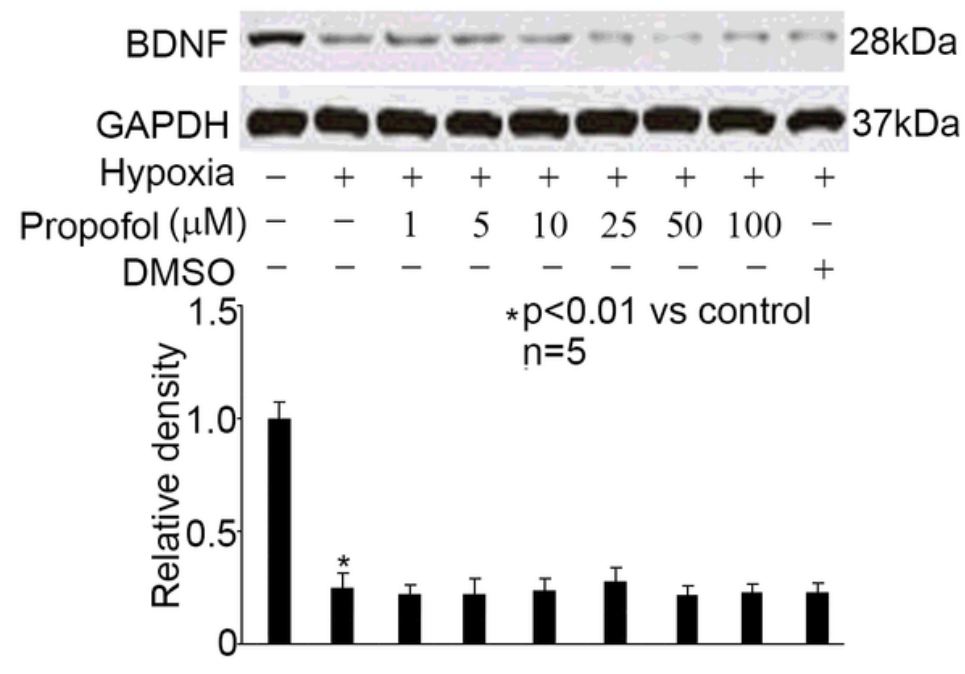

b
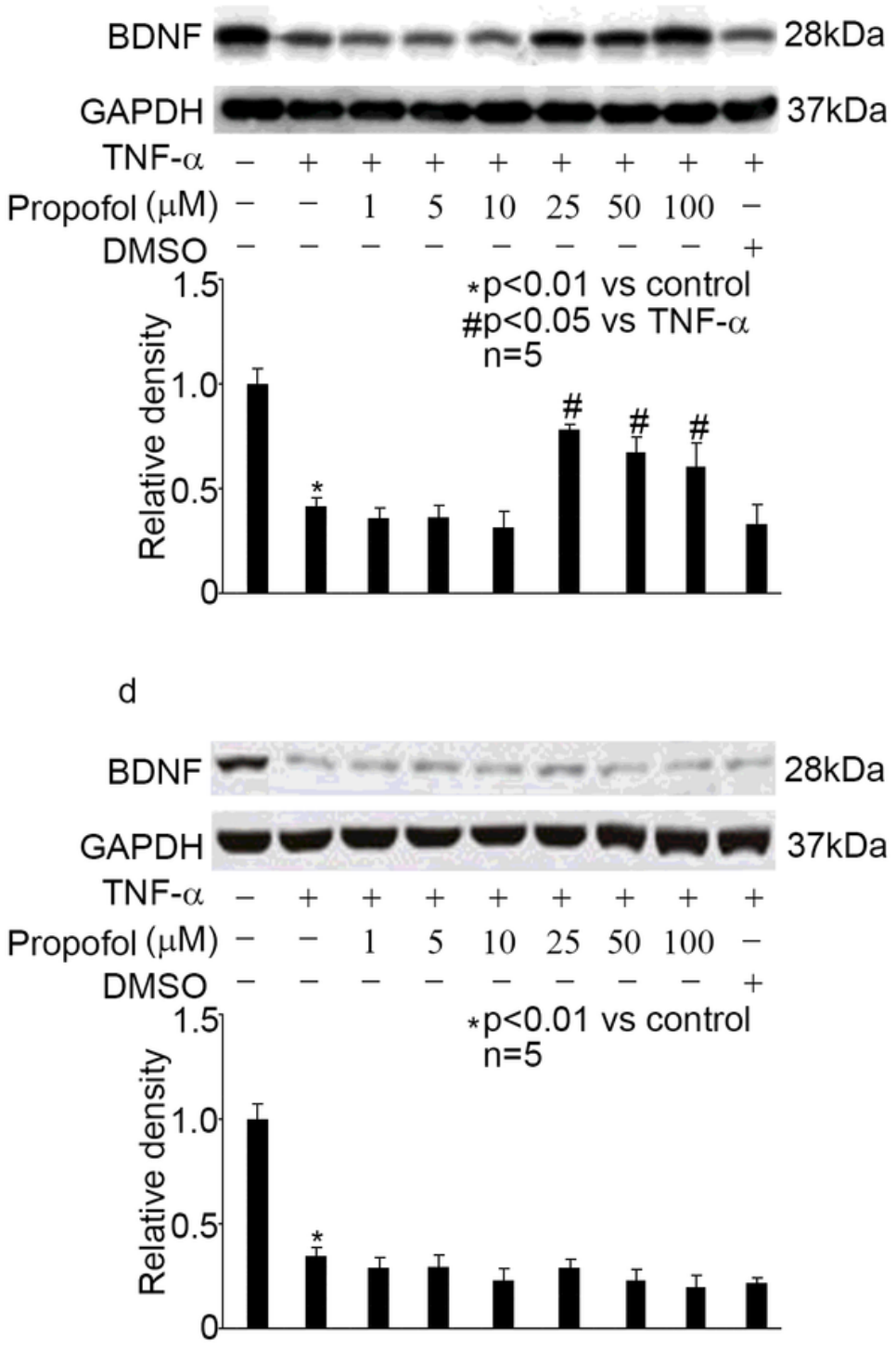

Figure 2

Propofol reversed hypoxia- and TNF-a-modulated BDNF reduction in rat hippocampal neurons. The upper panel was a representative experiment and the lower panel was the summary of densitometric data from 5 separate experiments. GAPDH served as loading control. Data were expressed as normalized ratio of protein band density of BDNF against GAPDH, and were presented as mean \pm standard deviation. (a) In hippocampal neurons, propofol induced BDNF production, which was inhibited by hypoxia $(5 \% 02,3 \mathrm{~h})$ treatment. (b) In hippocampal neurons, propofol induced BDNF production, which was inhibited by TNF-a 
(40ng/mL, 3h) treatment. (c) In astrocytes, propofol had no effect on hypoxia-modulated BDNF production. (d) In astrocytes, propofol had no effect on TNF-a-modulated BDNF production.

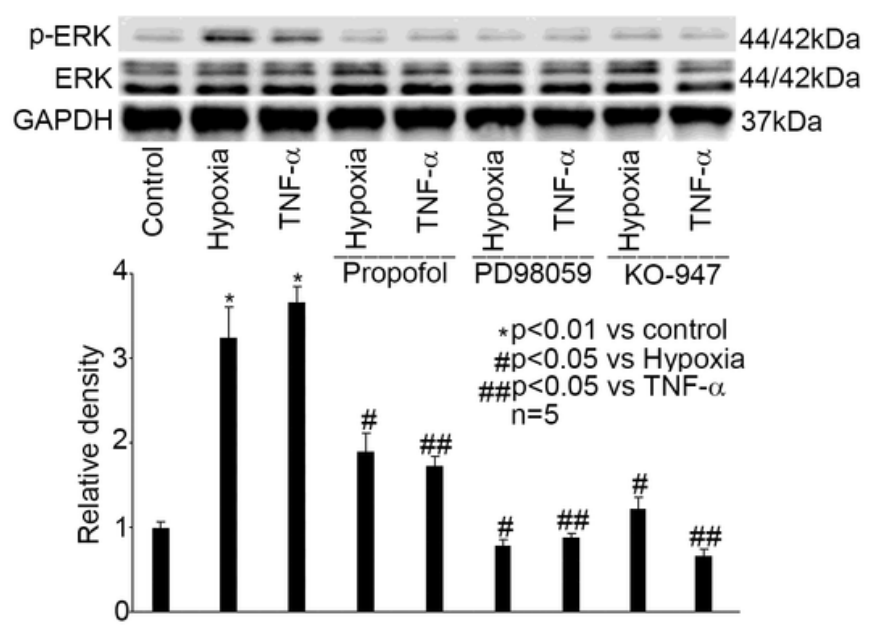

c

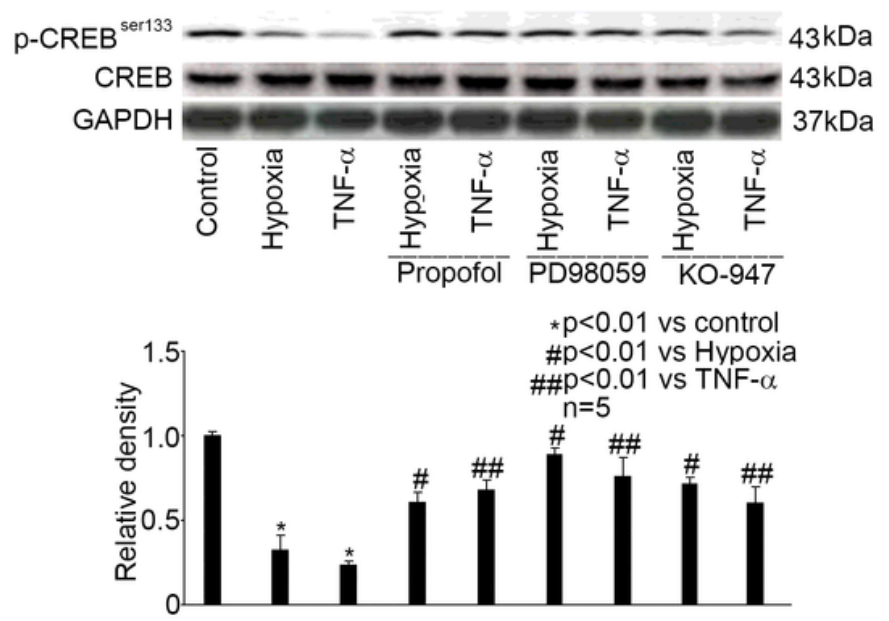

b

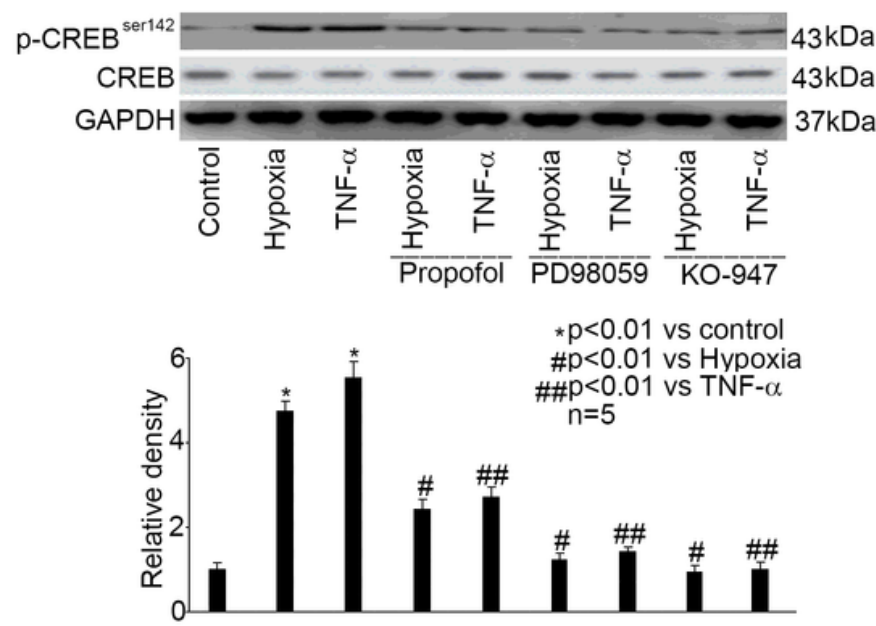

d
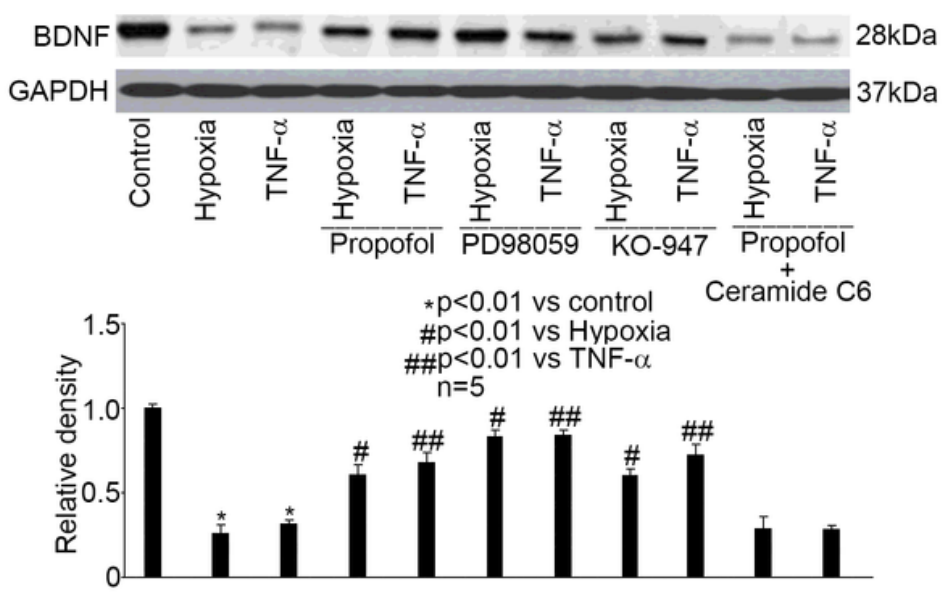

\section{Figure 3}

The beneficial effect of popofol on BDNF production was mediated through regulating the phosphorylation of ERK and CREB. (a) In rat hippocampal neurons, hypoxia ( $5 \% 02,3 \mathrm{~h}$ ) and TNF-a $(40 \mathrm{ng} / \mathrm{mL}, 3 \mathrm{~h})$ increased the phosphorylaion of ERK , which was attenuated by $25 \mu \mathrm{M}$ propofol, $10 \mu \mathrm{M}$ PD98059 or 10 $\mu \mathrm{M}$ K0-947. The upper panel was a representative experiment and the lower panel was the summary of densitometric data from 5 separate experiments. GAPDH served as loading control. Data were expressed as normalized ratio of protein band density of phosphorylated ERK against total ERK, which was normalized with GAPDH, and were presented as mean \pm standard deviation. (b) In rat hippocampal neurons, hypoxia ( $5 \% 02,3 \mathrm{~h})$ and TNF-a $(40 \mathrm{ng} / \mathrm{mL}, 3 \mathrm{~h})$ increased the phosphorylation of CREB at Ser142 (p-CREB Ser142), which was reversed by $25 \mu \mathrm{M}$ propofol, $10 \mu \mathrm{M}$ PD 98059 or $10 \mu \mathrm{M}$ KO- 
947. (c) In rat hippocampal neurons, hypoxia (5\% 02, 3h) and TNF-a $(40 \mathrm{ng} / \mathrm{mL}$, 3h) reduced the phosphorylation of CREB at Ser133 (p-CREB Ser133), which was reversed by $25 \mu \mathrm{M}$ propofol, $10 \mu \mathrm{M}$ PD98059 or $10 \mu \mathrm{M}$ KO-947. (d) In rat hippocampal neurons, hypoxia and TNF-a reduced BDNF production, which was reversed by $25 \mu \mathrm{M}$ propofol, $10 \mu \mathrm{M}$ PD 98059 or $10 \mu \mathrm{M}$ KO-947, and the beneficial effect of propofol on BDNF production was abolished by $10 \mu \mathrm{M}$ Ceramide C6.
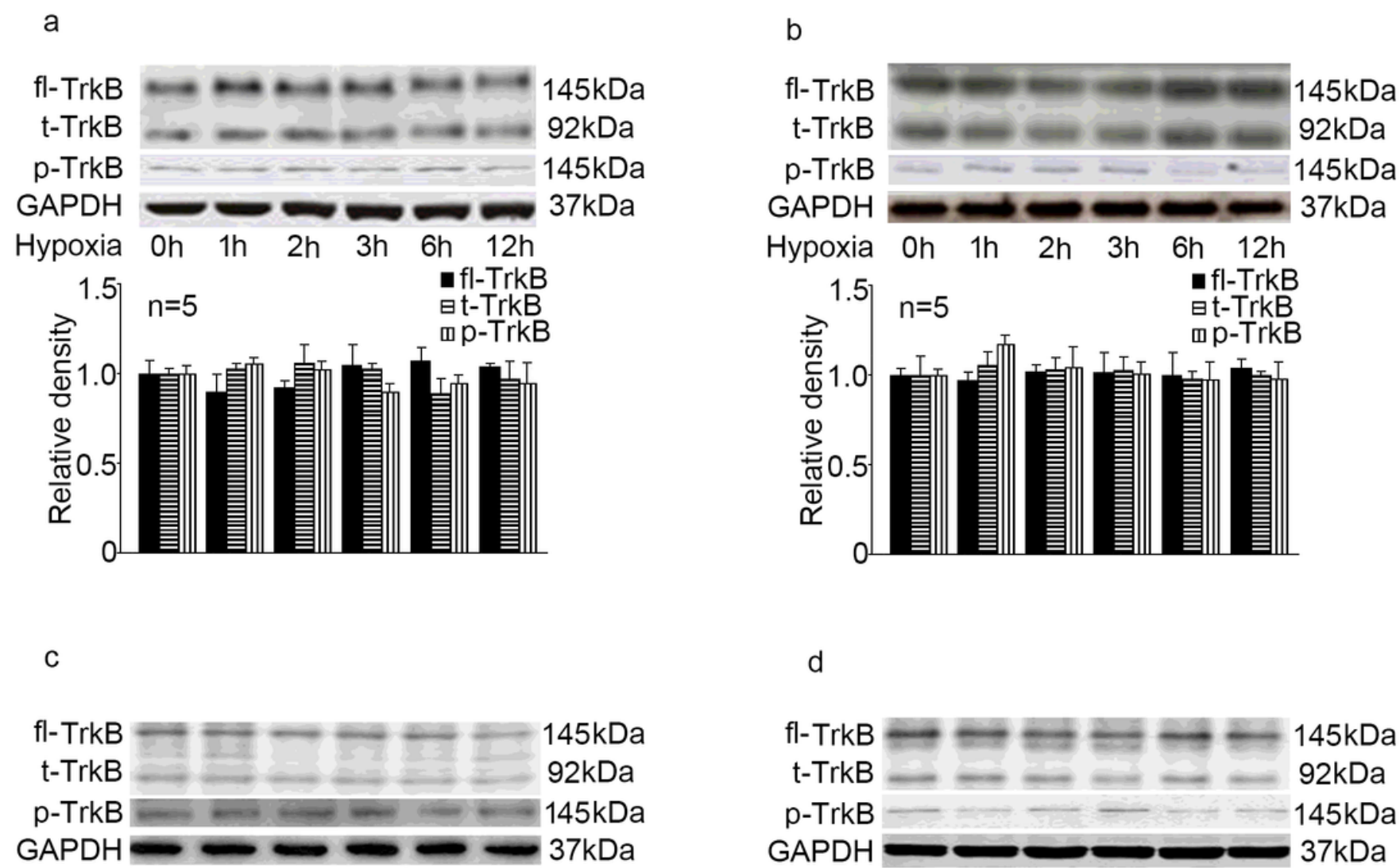

d

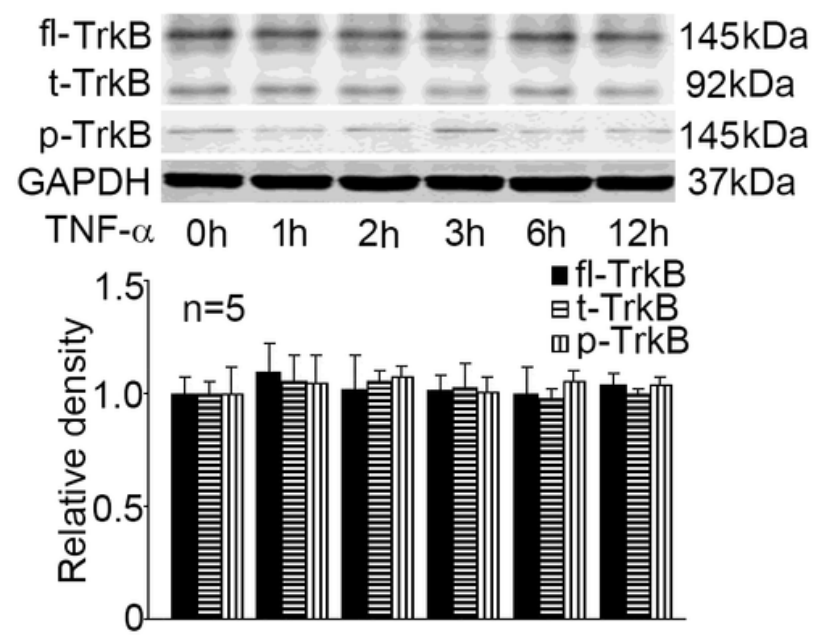

Figure 4

Hypoxia and TNF-a had no effect on full length TrkB (fl-TrkB) expression, truncation (t-TrkB) or phosphorylation ( $\mathrm{p}-\mathrm{TrkB})$ in rat hippocampal neurons and astrocytes. The upper panel was a representative experiment and the lower panel was the summary of densitometric data from 5 separate experiments. GAPDH served as loading control. Data were expressed as normalized ratio of protein band density of $\mathrm{p}-\mathrm{TrkB}$ or $\mathrm{t}$-TrkB against fl-TrkB, which was normalized with GAPDH, and were presented as mean \pm standard deviation. (a) In rat hippocampal neurons, hypoxia had no effect on the expression, 
truncation or phosphorylation of TrkB. (b) In astrocytes, hypoxia had no effect on the expression, truncation or phosphorylation of TrkB. (c) In rat hippocampal neurons, TNF-a had no effect on the expression, truncation or phosphorylation of TrkB. (d) In astrocytes, TNF-a had no effect on the expression, truncation or phosphorylation of TrkB.

a
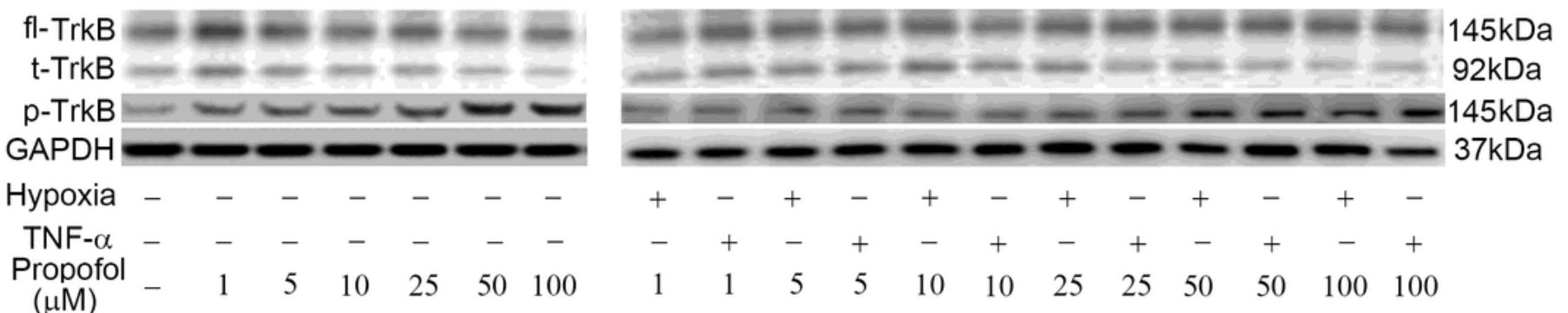

Hypoxia - $-\quad-\quad-\quad-\quad-$

TNF- $\alpha$ - $\quad-\quad-\quad-\quad-\quad-\quad-$

$\begin{array}{llllllll}\begin{array}{c}\text { Propofol } \\ (\mu \mathrm{M})\end{array} & - & 1 & 5 & 10 & 25 & 50 & 100\end{array}$

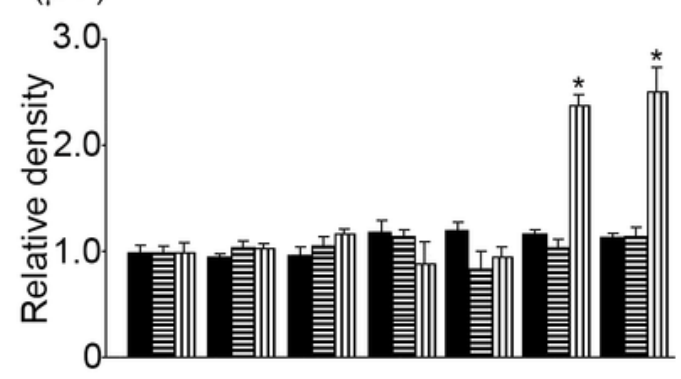

- fl-TrkB

घt-TrkB mp-TrkB $* p<0.01$ vs control $n=5$

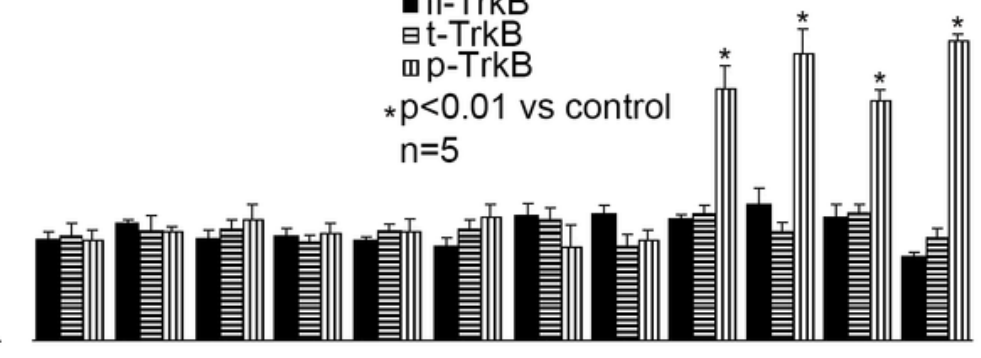

b
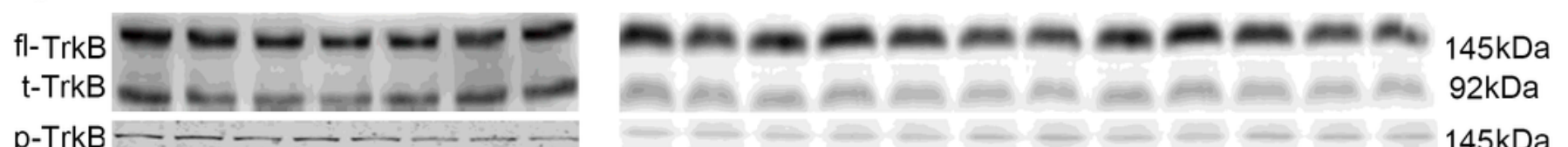

$\mathrm{p}$-TrkB $-\cdots+-\cdots$

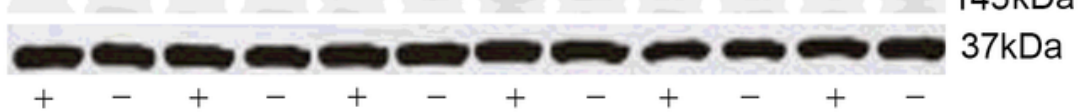

Hypoxia

$\begin{array}{llllllll}\begin{array}{c}\text { Propofol } \\ (\mu \mathrm{M})\end{array} & - & 1 & 5 & 10 & 25 & 50 & 100\end{array}$

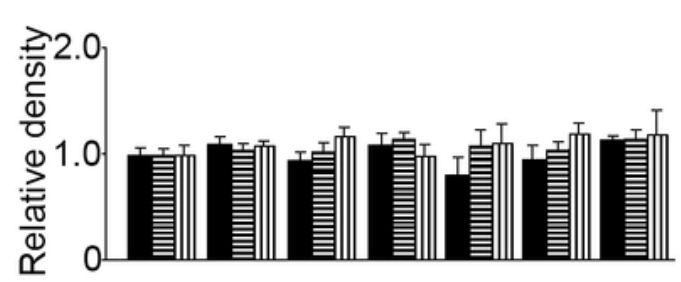

$\begin{array}{llllllllllll}1 & 1 & 5 & 5 & 10 & 10 & 25 & 25 & 50 & 50 & 100 & 100\end{array}$

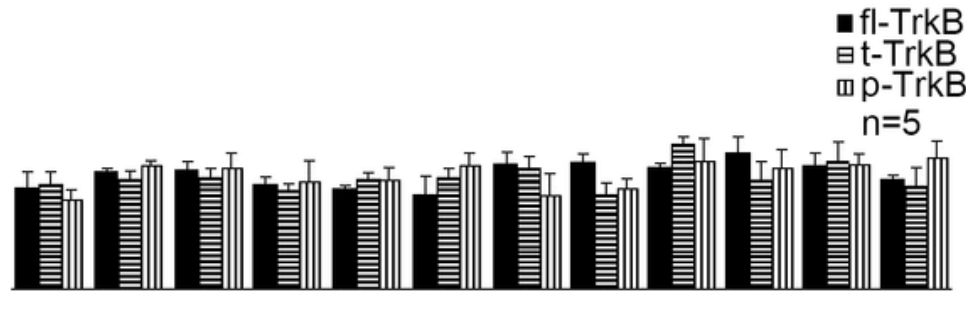

Figure 5 
Propofol induced TrkB phosphorylation in rat hippocampal neurons. The upper panel was a representative experiment and the lower panel was the summary of densitometric data from 5 separate experiments. GAPDH served as loading control. Data were expressed as normalized ratio of protein band density of $\mathrm{p}$-TrkB or t-TrkB against fl-TrkB, which was normalized with GAPDH, and were presented as mean \pm standard deviation. (a) In hippocampal neurons, propofol had no effect on TrkB expression or truncation, but induced TrkB phosphorylation. (b) In astrocytes, propofol had no effect on TrkB expression, truncation or phosphorylation (Figure $5 b$ ).

a

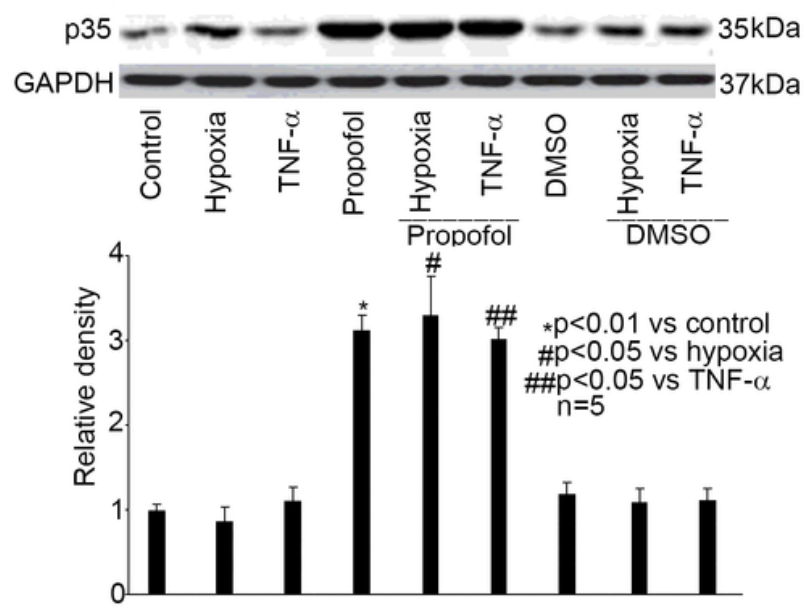

c

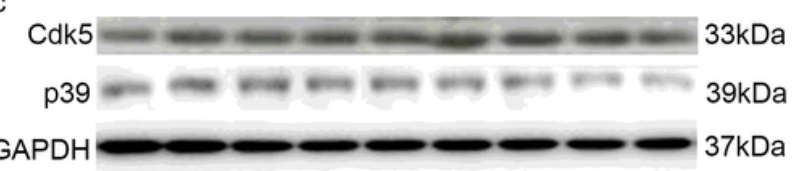

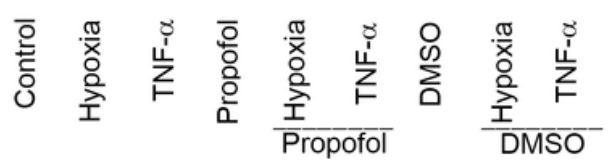

b

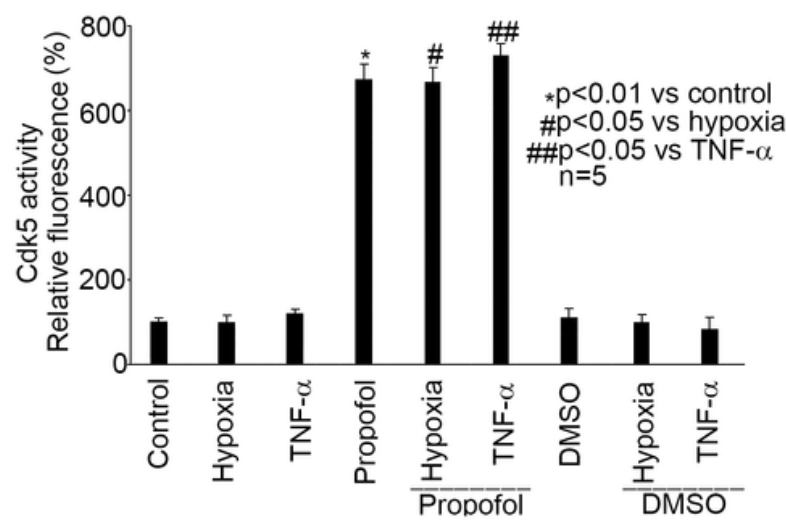

d p35 GAPDH
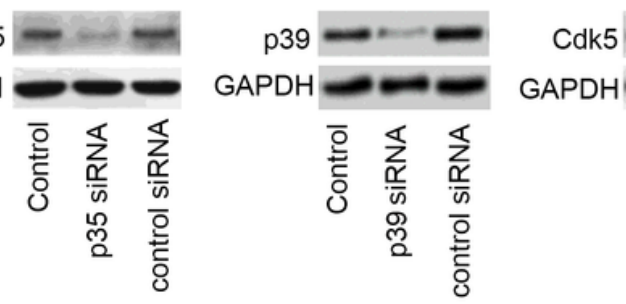

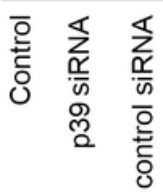
GAPDH

e
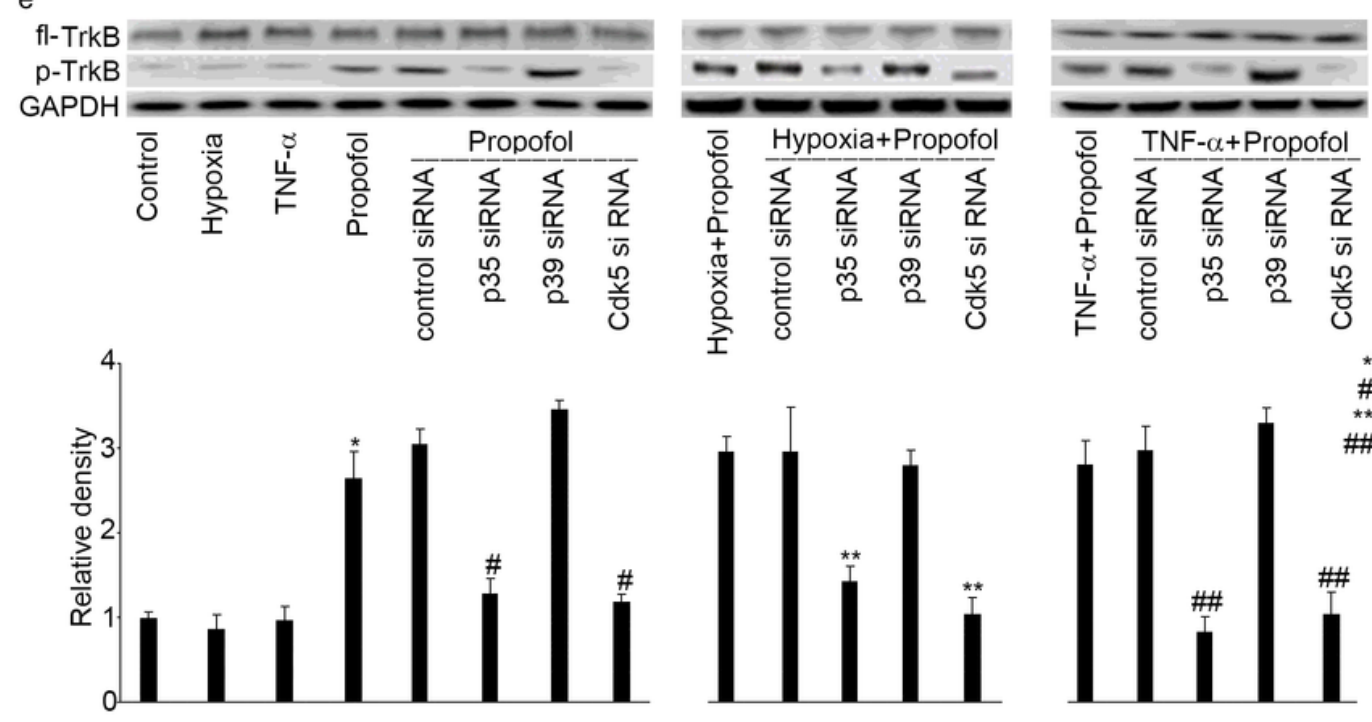
$* p<0.01$ vs control
$\# p<0.05$ vs Propofol $\# p<0.05$ vs Propofol
$* * p<0.05$ vs Hypoxia+Propofol II $\#$ \# $<0.05$ vs TNF- $\alpha+$ Propofol $\mathrm{n}=5$

Figure 6 
Propofol-induced TrkB phosphorylation was carried out via modulating p35 expression and Cdk5activation in hippocampal neurons. (a) Propofol induced the expression of p35. The upper panel was a representative experiment and the lower panel was the summary of densitometric data from 5 separate experiments. GAPDH served as loading control. Data were expressed as normalized ratio of protein band density of p35 against GAPDH, and were presented as mean \pm standard deviation. (b) Propofol induced the activation of Cdk5. Data were expressed as relative fluorescence compared with that of untreated control cells, and were presented as mean \pm standard deviation. $100 \%$ activity was set for control cells. (c) Propofol had no effect on the expression of Cdk5 and p39. The panel was a representative experiment, and GAPDH served as loading control. (d) Transfection efficiency of siRNAs against p35, p39 and Cdk5 were evaluated by Western blot. Untransfected neurons served as normal control, and control siRNA-transfected neurons served as transfection control. The panel was a representative experiment. (e) Transfection of siRNA against p35 or Cdk5 alleviated propofol-induced TrkB phosphorylation, while transfection of siRNA against $\mathrm{p} 39$ had no such effect. The upper panel was a representative experiment and the lower panel was the summary of densitometric data from 5 separate experiments. GAPDH served as loading control. Data were expressed as normalized ratio of protein band density of $\mathrm{p}$-TrkB against fl-TrkB, which was normalized with GAPDH, and were presented as mean \pm standard deviation. 